\title{
Generation of Walsh codes in two different orderings using 4-bit Gray and Inverse Gray codes
}

\author{
K.Usha ${ }^{1^{*}}$ and K. Jaya Sankar ${ }^{2}$ \\ ${ }^{1 *}$ ECE Department, MVSR Engineering College, Hyderabad - 501510, India \\ ${ }^{2}$ ECE Department, Vasavi College of Engineering, Hyderabad-500031, India \\ u_kamle@yahoo.com*, kottareddyjs@gmail.com
}

\begin{abstract}
Walsh code sequences are fixed power codes and are widely used in multi-user CDMA communications. Walsh code is a group of spreading codes having good autocorrelation properties and poor cross-correlation properties. This paper presents a simple technique to construct Walsh code sets of any length recursively using 4-bit Gray and Inverse Gray codes. An n-bit Gray code is a list of all $2^{n}$ bit strings such that adjacent code words in the sequence differ in only one bit position. An ' $n$ ' bit Inverse Gray code , is defined exactly opposite to Gray code, it is a list of all $2^{\text {n }}$ bit strings of length ' $n$ ' each, such that successive code words differ in ( $n-1)$ bit positions. If the first and last code words also differ in one bit position then the resultant code is called cyclic. The technique presented in this paper allows us to construct 4 ! Walsh code set (of any length) orderings since they are constructed from 4-bit Gray and Inverse Gray codes. All these Walsh code sets are not symmetrical along rows and columns. A Gray-Binary mapping technique is adopted to transform these Walsh code sets into symmetrical matrices. n-bit Gray codes are used for mapping $2^{n}$-length Walsh code sets. Out of $n$ ! permutations few result in equal row column transition counts. And two permutations transform these Walsh code sets into Walsh-Hadamard and Walsh-Paley sequence orderings.
\end{abstract}

Keywords: Gray code, Inverse Gray code, Walsh code, Walsh-Hadamard matrix, Walsh-Paley matrix, Row Transition Count, Column transition count.

Introduction

Walsh codes are fixed length orthogonal codes possessing high auto correlation and low cross correlation properties. Walsh codes are linear phase and zero mean with unique number of zero crossings for each sequence within the set. Walsh codes are commonly used as spreading sequences in Direct Sequence Spread Spectrum (DS-SS) communications. Walsh codes are the backbone of CDMA systems and are used to develop the individual channels in CDMA (Harmuth, 1969; Beauchamp, 1975). Channelization by means of code multiplexing is a fundamental feature of IS-95 systems. In particular, channelization is accomplished using length64 Walsh codes, which are assigned to different channels. Walsh codes can be generated recursively and indexed according to their row number in the $M$ by $M$ Walsh matrix. Their popularity is due to the ease of implementation. Among the former techniques for the generation of Walsh codes the popular method is based on the simple iterative scheme from the Hadamard matrices. This paper presents a technique for the construction of Walsh codes using Gray and Inverse Gray codes. And then using a mapping technique rearrangement of these code words is done to result in equal row and column bit transition counts. This mapping technique allows us to generate Walsh-Hadamard and Walsh-Paley matrices. Walsh code set in dyadic Paley ordering is also known as Gray code Sequence ordering. In CDMA, Walsh-Hadamard matrices are used for user separation. The $\mathrm{M} X \mathrm{M}$ Walsh-Hadamard $(\mathrm{WH})$ matrices $H_{M}$, where $M$ is a power of two, are defined by $H_{1}=1$ and the recursive relation

$$
\mathrm{H}_{\mathrm{M}}=\left[\begin{array}{l}
\mathrm{H}_{\mathrm{M}} / 2 \mathrm{H}_{\mathrm{M}} / 2 \\
\mathrm{H}_{\mathrm{M}} / 2-\mathrm{H}_{\mathrm{M} / 2}
\end{array}\right]
$$

For example, the matrix $\mathrm{H}_{8}$ is given by

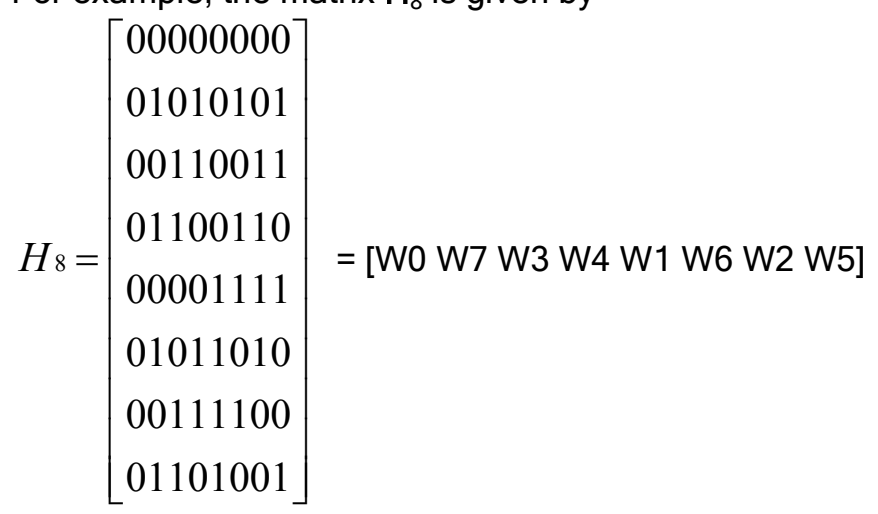

Walsh -Paley matrix of order three is given by

$$
W P(3)=\left[\begin{array}{l}
00000000 \\
00001111 \\
00110011 \\
00111100 \\
01010101 \\
01011010 \\
01100110 \\
01101001
\end{array}\right]=\left[\begin{array}{c}
\mathrm{W} 0 \\
\mathrm{~W} 1 \\
\mathrm{~W} 3 \\
\mathrm{~W} 2 \\
\mathrm{~W} 7 \\
\mathrm{~W} 6 \\
\mathrm{~W} 4 \\
\mathrm{~W} 5
\end{array}\right]
$$

An n-bit Gray code is a list of all $2^{n}$ bit strings such that successive code words differ in only one bit position. 
If the first and last code words also differ in one bit position then the resultant code is called cyclic. Gray codes have the adjacency property which makes the Hamming distance between adjacent code words always equal to 1. Inverse Gray codes, as defined in (Duc-Minh Pham et al., 2011), on the contrary, exhibit maximum possible Hamming distance $(n-1)$ between the two successive code words. A very commonly used method of generating $\mathrm{n}$-bit Gray code from binary is by performing bit-wise XOR operation of two successive bits. In (Falkowski \& Sasao, 2005), generation of Walsh functions in four different orderings from primary Rademacher functions is discussed.

The paper is organized in the following manner: Section II briefly discusses the Binary Cyclic Gray (Jaya Sankar, 2004) and Inverse Gray code generation algorithm. The procedure for the construction of Walsh code sets from 4-bit Gray and Inverse Gray codes is explained in Section-III. In Section -IV the re-arrangement of Walsh code words using the mapping technique (Doran, 2007) to form different sequence orderings such as Walsh-Hadamard, Walsh-Paley matrices is discussed. And finally, Section- $V$ concludes the paper with future work.

\section{Algorithm to generate binary cyclic Gray and Inverse Gray codes}

Let $\left(P_{1}, P_{2}, P_{3}, \ldots \ldots \ldots . P_{n}\right)$ be a permutation of $(1,2,3, \ldots . n)$. The $M=2^{n}$ integers $\left(0,1,2, \ldots . .,\left(2^{n}-1\right)\right)$ can be arranged in the following indexed indicial sets.

$Q_{0}=2^{0}\{1,3,5 \ldots \ldots\}$

$\mathrm{Q}_{1}=2^{1}\{1,3,5 \ldots \ldots\}$

$Q_{n-1}=2^{n-1}$

$Q_{n}=2^{n}$

$\mathrm{n}$ - bit Cyclic Gray code, $\mathrm{M}=2^{\mathrm{n}}$.

Let an n-bit Cyclic Gray code be needed. Then, starting with the row of all zeros as a zeroeth row, the $i^{\text {th }}$ row is obtained from the $(i-1)^{\text {th }}$ row by replacing the $P_{j}^{\text {th }}$ bit by its successor, if it is in $Q_{j-1}$.

Let us consider the construction of a 4 -bit binary Gray code. All the integers, i.e., $\left\{0,1,2,3, \ldots . .\left(2^{3}-1\right)\right\}$ are arranged in the form of indicial sets as shown below:

$Q_{0}=2^{0}\{1,3,5,7,9,11,13,15\}=1,3,5,7,9,11,13,15$

$Q_{1}=2^{1}\{1,3,5,7\}=2,6,10,14$

$Q_{2}=2^{2}\{1,3\}=4,12$

$\mathrm{Q}_{3}=2^{3}\{1\}=8$

As stated earlier, let $\left(P_{1}, P_{2}, P_{3} \ldots . . P_{j} \ldots . . . P_{n}\right)$ be a permutation of $(1,2,3, \ldots . j, \ldots . . n)$. Since we are considering a 4-bit case, consider the permutation $\{3,4,1,2\}$. Hence, $P_{1}=3 ; P_{2}=4 ; P_{3}=1 ; P_{4}=2$. The first code word is $(000$ $0)$ which is the zeroeth row of the code. To obtain $1^{\text {st }}$ row, we have to change $P_{j}{ }^{\text {th }}$ bit if ' 1 ' is in $Q_{j-1} \cdot$ Here, 1 is in $Q_{0}$. Therefore, $P_{1}$ bit is to be changed and $P_{1}=3$, hence the

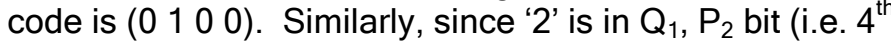
bit) is to be changed, hence the code is $\left(\begin{array}{llll}1 & 1 & 0 & 0\end{array}\right)$. The resulting code obtained by continuing this procedure is tabulated in Table I.

$\mathrm{n}$ - bit Cyclic Inverse Gray code, $M=2^{n}$.

The procedure to generate n-bit Inverse Gray codes is exactly opposite to Gray code generation. Let an n-bit Cyclic Inverse Gray code be needed. Then, for ' $n$ ' even, starting with the row of all zeros as a zeroeth row, the $i^{\text {th }}$ row is obtained from the $(\mathrm{i}-1)^{\text {th }}$ row by complementing all other bits except the $P_{j}^{\text {th }}$ bit by its successor, if it is in $Q_{j-1}$. And for ' $n$ ' odd, the above procedure is used to obtain all the rows except $M / 2$ th row. For $M / 2$ th row, all the bits have to changed irrespective of where it falls within the indicial sets.

Let us consider the construction of a 4-bit Inverse Gray code. As stated earlier, let $\left(P_{1}, P_{2}, P_{3} \ldots . . . P_{j} \ldots . . . P_{k}\right)$ be a permutation of $(1,2,3, \ldots . j, \ldots . n)$. Since we are considering a 4-bit case, consider the permutation $\{3,4,1,2\}$. Hence, $P_{1}=3 ; P_{2}=4 ; P_{3}=1 ; P_{4}=2$. The first

Table 1. A 4-bit Cyclic Gray and Inverse Gray code generation with permutation $\{3,4,1,2\}$

\begin{tabular}{|c|c|c|c|c|c|c|c|c|}
\hline Element No & $\mathrm{i}^{\text {th }}$ row & $P_{j}$ & $\begin{array}{l}\text { Bit to be } \\
\text { Changed } \\
\text { to obtain } \\
\text { Gray code }\end{array}$ & $\begin{array}{c}\text { 4-bit } \\
\text { Gray code } \\
43221\end{array}$ & $\begin{array}{l}\text { 4-bit Gray } \\
\text { code in } \\
\text { Decimal } \\
\text { notation }\end{array}$ & $\begin{array}{c}\text { Bits to be } \\
\text { Changed to } \\
\text { obtain Inverse } \\
\text { Gray code }\end{array}$ & $\begin{array}{c}\text { 4-bit } \\
\text { Inverse } \\
\text { Gray code } \\
43221 \\
\end{array}$ & $\begin{array}{c}\text { 4-bit Inverse } \\
\text { Gray code in } \\
\text { Decimal } \\
\text { notation }\end{array}$ \\
\hline 1 & 0 & - & - & $\begin{array}{lllll}0 & 0 & 0 & 0\end{array}$ & 0 & - & 0000 & 0 \\
\hline 2 & 1 & $\mathrm{P}_{1}$ & 3 & $0 \begin{array}{llll}0 & 1 & 0 & 0\end{array}$ & 4 & $1,2,4$ & $\begin{array}{llll}1 & 0 & 1 & 1\end{array}$ & 11 \\
\hline 3 & 2 & $\mathrm{P}_{2}$ & 4 & 111000 & 12 & $1,2,3$ & 11100 & 12 \\
\hline 4 & 3 & $\mathrm{P}_{1}$ & 3 & 100000 & 8 & $1,2,4$ & $\begin{array}{llll}0 & 1 & 1 & 1 \\
\end{array}$ & 7 \\
\hline 5 & 4 & $\mathrm{P}_{3}$ & 1 & 100001 & 9 & $2,3,4$ & 100001 & 9 \\
\hline 6 & 5 & $\mathrm{P}_{1}$ & 3 & 111001 & 13 & $1,2,4$ & $0 \begin{array}{llll}0 & 0 & 1 & 0 \\
\end{array}$ & 2 \\
\hline 7 & 6 & $\mathrm{P}_{2}$ & 4 & $\begin{array}{llll}0 & 1 & 0 & 1 \\
\end{array}$ & 5 & $1,2,3$ & $\begin{array}{llll}0 & 1 & 0 & 1 \\
\end{array}$ & 5 \\
\hline 8 & 7 & $\mathrm{P}_{1}$ & 3 & $\begin{array}{llll}0 & 0 & 0 & 1 \\
\end{array}$ & 1 & $1,2,4$ & 1110 & 14 \\
\hline 9 & 8 & $\mathrm{P}_{4}$ & 2 & $\begin{array}{llll}0 & 0 & 1 & 1 \\
\end{array}$ & 3 & $1,3,4$ & $\begin{array}{llll}0 & 0 & 1 & 1 \\
\end{array}$ & 3 \\
\hline 10 & 9 & $\mathrm{P}_{1}$ & 3 & $\begin{array}{llll}0 & 1 & 11 \\
\end{array}$ & 7 & $1,2,4$ & 100000 & 8 \\
\hline 11 & 10 & $\mathrm{P}_{2}$ & 4 & 11111 & 15 & $1,2,3$ & $\begin{array}{llll}1 & 1 & 1 & 1 \\
\end{array}$ & 15 \\
\hline 12 & 11 & $\mathrm{P}_{1}$ & 3 & $\begin{array}{llll}1 & 0 & 1 & 1 \\
\end{array}$ & 11 & $1,2,4$ & $\begin{array}{llll}0 & 1 & 0 & 0 \\
\end{array}$ & 4 \\
\hline 13 & 12 & $\mathrm{P}_{3}$ & 1 & 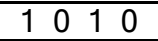 & 10 & $2,3,4$ & 100110 & 10 \\
\hline 14 & 13 & $\mathrm{P}_{1}$ & 3 & 1110 & 14 & $1,2,4$ & $\begin{array}{llll}0 & 0 & 0 & 1 \\
\end{array}$ & 1 \\
\hline 15 & 14 & $\mathrm{P}_{2}$ & 4 & $\begin{array}{llll}0 & 1 & 1 & 0 \\
\end{array}$ & 6 & $1,2,3$ & $\begin{array}{llll}0 & 1 & 1 & 0 \\
\end{array}$ & 6 \\
\hline 16 & 15 & $\mathrm{P}_{1}$ & 3 & $0 \begin{array}{llll}0 & 0 & 1 & 0\end{array}$ & 2 & $1,2,4$ & 111001 & 13 \\
\hline $\begin{array}{l}\text { Research article } \\
\text { (CIndian Society f }\end{array}$ & ion & $m$ & & http://ww & $\begin{array}{l}\text { le" } \\
\text { st.org }\end{array}$ & & & $\begin{array}{l}\text { ha \& Jaya San } \\
\text { Indian J.Sci.Techr }\end{array}$ \\
\hline
\end{tabular}


code word is $\left(\begin{array}{lll}0 & 0 & 0\end{array}\right)$ which is the zeroeth row of the code. To obtain $1^{\text {st }}$ row, we have to change all other bits except $P_{j}^{\text {th }}$ bit if ' 1 ' is in $Q_{j-1}$. Here, 1 is in $Q_{0}$. Therefore, retaining $P_{1}$ bit as it is all other bits are to be changed, since $P_{1}=3,3^{\text {rd }}$ bit is retained and $1^{\text {st }}, 2^{\text {nd }}$ and $4^{\text {th }}$ bits are changed. Hence the resultant codeword of the $1^{\text {st }}$ row is (1 $\left.\begin{array}{llll}0 & 1 & 1\end{array}\right)$. Similarly, to obtain $2^{\text {nd }}$ row since ' 2 ' is in $Q_{1}, P_{2}$ bit is to be unchanged, hence the code is $\left(\begin{array}{lll}1 & 1 & 0\end{array}\right)$. Table 1shows the resulting code obtained by continuing this procedure.

Using the above algorithm, 4! i.e. 24 possible combinations of 4-bit binary Inverse Gray codes can be generated. A total of $n$ ! Gray and Inverse Gray codes can be generated using the above technique for any integer value of ' $n$ ' and all these codes are cyclic.

\section{Construction of Walsh codes}

Walsh code is defined as a group of $2 \mathrm{M}$ code words which (Walsh, 1923) contain 2M binary elements which with themselves and their logical Inverses form a mutually orthogonal set. Walsh code is known popularly as a group of spreading codes having good autocorrelation properties and poor cross-correlation properties. Walsh codes are the backbone of CDMA systems and are used to develop the individual channels in CDMA (Harmuth, 1969; Beauchamp, 1975). The method explained in this section allows us to recursively construct Walsh codes of any length.

Table 2. A 8-length Walsh code set generated using the permutation $\{1,2,3,4\}$.

\begin{tabular}{|l|l|l|}
\hline $\begin{array}{l}\text { 4-bit } \\
\text { Gray code }\end{array}$ & $\begin{array}{l}\text { 4-bit Inverse } \\
\text { Gray code }\end{array}$ & $\begin{array}{l}\text { Combined } \\
8 \text {-bit code }\end{array}$ \\
\hline 0000 & 0000 & 00000000 \\
0001 & 1110 & 00011110 \\
0011 & 0011 & 00110011 \\
0010 & 1101 & 00101101 \\
0110 & 0110 & 01100110 \\
0111 & 1000 & 01111000 \\
0101 & 0101 & 01010101 \\
0100 & 1011 & 01001011 \\
1100 & 1100 & 11001100 \\
1101 & 0010 & 11010010 \\
1111 & 1111 & 11111111 \\
1110 & 0001 & 11100001 \\
1010 & 1010 & 10101010 \\
1011 & 0100 & 10110100 \\
1001 & 1001 & 10011001 \\
1000 & 0111 & 10000111 \\
\hline
\end{tabular}

\section{8-length Walsh code set construction}

During the initial investigation to construct 8-length Walsh code set using Gray and Inverse Gray codes with different ' $n$ ' values only $n=4$ came up with good results as $M=n+n$. A combined 8-bit $(4+4)$ code of $16\left(2^{4}\right)$ code words is constructed by appending 4-bit Gray code with 4-bit Inverse Gray code generated using the algorithms detailed in section II \& III for a chosen permutation. Each column in the top-half of this combined code is a 8-length Walsh code word. And the bottom-half is the dual or logical Inverse of top-half. Table 2 gives the 8-length Walsh code set generated using the permutation $\{1,2,3$,
4\}. With 4! Permutations, 24 different sequence orderings of 8-length Walsh code set is obtained.

\section{6-length Walsh code set Construction}

If the 8-bit combined code is appended to itself by retaining the top-half as it is and complementing the bottom-half then all the 16 columns result in a 16-length Walsh code set. Table 3 gives the 16- length Walsh code set obtained from the above said procedure. The Bold faced code words are the ones, which are appended after complementing. This 16 -length Walsh code set is used as a basic set for generating larger sets. Walsh code sets of length $32,64,128, \ldots$ can be constructed recursively using the following relationship

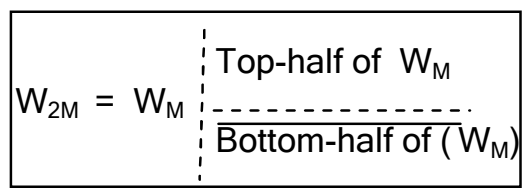

Table 3. A 16-length Walsh code set

\begin{tabular}{|c|}
\hline $\begin{array}{c}\text { Combined 8-bit code appended to itself by } \\
\text { complementing bottom-half }\end{array}$ \\
\hline $0000000 \mid 00000000$ \\
$0001111 \mid 00011110$ \\
$0011001 \mid 00110011$ \\
$010110 \mid 00101101$ \\
$0110011 \mid 01100110$ \\
$0101010 \mid 01111000$ \\
$0100101 \mid 01010101$ \\
$1100110 \mid 00110011$ \\
$1101001 \mid 00101101$ \\
$1111111 \mid 00000000$ \\
$1110000 \mid 00011110$ \\
$1010101 \mid 01010101$ \\
$1011010 \mid 01001011$ \\
$1001100 \mid 01100110$ \\
$1000011 \mid 01111000$ \\
\hline
\end{tabular}

The logical inverses of the Walsh code words of lengths $8,16,32$ in decimal notation obtained using permutation $\{1,3,4,2\}$ are tabulated in Table 4 .

Gray-Binary Mapping Technique to generate Walsh codes in two different sequence orderings

The Walsh code sets constructed using the above said procedure do not observe symmetry along rows and columns i.e. they do not have equal bit transitions along the rows and columns. Walsh-Hadamard and Walsh Paley matrices have a predefined sequence order and are symmetrical along rows and columns. So, to obtain the symmetry a mapping technique (Doran 2007) is used. First, an n-bit Gray code is generated using the algorithm in Section-II. Next, each code word is assigned a Gray rank. 'Gray rank' is the position of the generated n-bit Gray code word with reference to the natural binary sequence order. Then, rearrangement of the columns (with different bit transitions) of the constructed M-length $\left(M=2^{n}\right)$ Walsh code sets is done according to the Gray ranking. And it is observed that only a few permutations 
Table 4. The logical inverses of the Walsh code words of lengths 8, 16, 32 in decimal notation obtained using

\begin{tabular}{|c|c|c|}
\hline $\begin{array}{l}\text { 8-length } \\
\text { Walsh code } \\
\text { set }\end{array}$ & $\begin{array}{c}16 \text {-length } \\
\text { Walsh code set }\end{array}$ & $\begin{array}{c}\text { 32-length } \\
\text { Walsh code set }\end{array}$ \\
\hline $\begin{array}{l}255 \\
195 \\
240 \\
153 \\
170 \\
150 \\
165 \\
204\end{array}$ & $\begin{array}{l}65280 \\
50115 \\
61455 \\
39321 \\
43605 \\
38550 \\
42330 \\
52428 \\
65535 \\
49980 \\
61680 \\
39270 \\
43690 \\
38505 \\
42405 \\
52275\end{array}$ & $\begin{array}{l}4278255360 \\
3284386755 \\
4027576335 \\
2576980377 \\
2857740885 \\
2526451350 \\
2774181210 \\
3435973836 \\
4294967295 \\
3275539260 \\
4042322160 \\
2573637990 \\
2863311530 \\
2523502185 \\
2779096485 \\
3425946675 \\
4278190335 \\
3284352060 \\
4027518960 \\
2576967270 \\
2857719210 \\
2526439785 \\
2774162085 \\
3435934515 \\
4294901760 \\
3275504835 \\
4042264335 \\
2573624985 \\
2863289685 \\
2523490710 \\
2779077210 \\
3425907660\end{array}$ \\
\hline
\end{tabular}

out of $n$ ! result in symmetry. This method of mapping for 8-length Walsh code set with two different permutations along with the row and column transition counts is given in Table 5. For $n=3(M=8)$, four permutations out of six (n!) resulted in symmetrical row and column transition counts $\left(\mathrm{TC}_{\mathrm{R}}=\mathrm{TC}_{\mathrm{C}}\right)$. These permutations are $\{1,2,3\}$, $\{2,3,1\},\{3,1,2\}$ and $\{3,2,1\}$. Out of these four permutations two permutations resulted in Walsh-Hadamard and Walsh-Paley sequence ordering. Walsh-Hadamard matrix of order 3 is obtained with the permutation $\{3,2,1\}$ and Walsh-Paley matrix of order 3 is obtained with the permutation $\{1,2,3\}$. Number of permutations which resulted in symmetrical matrices for a given value of ' $n$ ' is denoted as Permutations with Equal Row, Column transition Counts $\left(\mathrm{PERCC}_{n}\right)$. Same procedure of obtaining symmetry is extended to $n=4,5 \& 6$ to rearrange the columns of $16,32 \& 64$ length Walsh code sets respectively and the results are given in Table 6 . PERCC $_{n}$ is observed to be following a specific pattern and is given by PERCC $_{n}=(n-1)$ PERCC $_{n-2}+$ PERCC $_{n-1}$

Table 7 \& Table 8 show the transformation of 8length Walsh code set generated using the permutation $\{1,2,3,4\}$ into Walsh - Hadamard and Walsh-Paley Sequence orderings respectively. Similarly 16-length Walsh code set also can be transformed into WalshHadamard and Walsh-Paley orderings by adopting the mapping technique with the permutations $\{1,2,3,4\}$ and $\{4,3,2,1\}$. The same can be extended to Walsh code set of any length.

Conclusion

Walsh code sets of any length can be constructed using the proposed technique. This technique allows us to construct 4 ! Walsh code set (of any length) orderings since they are constructed from 4-bit Gray and Inverse Gray codes. All these Walsh code sets are not symmetrical along rows and columns. Symmetry along

Table 5. Mapping for 8-length Walsh code set with two different permutations along with the row and column transition counts

\begin{tabular}{|c|c|c|c|c|c|}
\hline $\begin{array}{c}\text { 3-bit Gray code } \\
\text { Using permutation } \\
\{1,3,2\}\end{array}$ & $\begin{array}{l}\text { Natural Binary } \\
\text { Sequence Order }\end{array}$ & Gray rank & $\begin{array}{c}\text { Rearranged } \\
\text { 8-length } \\
\text { Walsh code set }\end{array}$ & $\begin{array}{l}\text { Row Transition } \\
\text { Count }\left(\mathrm{TC}_{\mathrm{R}}\right)\end{array}$ & $\begin{array}{l}\text { Column Transition Count } \\
\text { ( }\left(\mathrm{TC}_{\mathrm{C}}\right)\end{array}$ \\
\hline 000 & 0 & 0 & 00000000 & 0 & 0 \\
\hline 001 & 1 & 1 & 00110011 & 3 & 1 \\
\hline 101 & 2 & 7 & 00001111 & 1 & 7 \\
\hline 100 & 3 & 6 & 00111100 & 2 & 6 \\
\hline 110 & 4 & 3 & 01010101 & 7 & 3 \\
\hline 111 & 5 & 2 & 01100110 & 4 & 2 \\
\hline 011 & 6 & 4 & 01011010 & 6 & 4 \\
\hline 010 & 7 & 5 & 01101001 & 5 & 5 \\
\hline \multicolumn{6}{|c|}{ 3-bit Gray code Using permutation $\{2,3,1\}$} \\
\hline 000 & 0 & 0 & 00000000 & 0 & 0 \\
\hline 010 & 1 & 7 & 01010101 & 7 & 7 \\
\hline 110 & 2 & 1 & 00001111 & 1 & 1 \\
\hline 100 & 3 & 6 & 01011010 & 6 & 6 \\
\hline 101 & 4 & 3 & 00110011 & 3 & 3 \\
\hline 111 & 5 & 4 & 01100110 & 4 & 4 \\
\hline 011 & 6 & 2 & 00111100 & 2 & 2 \\
\hline 001 & 7 & 5 & 01101001 & 5 & 5 \\
\hline
\end{tabular}


Table 6. Permutations with Equal Row, Column transition Counts for 16, 32 \& 64 length Walsh code sets

\begin{tabular}{|l|l|l|l|}
\hline $\mathrm{n}$ & $\mathrm{n} !$ & $\begin{array}{l}\text { Walsh code set } \\
\text { of length } \mathrm{M}=2^{\mathrm{n}}\end{array}$ & PERCC $_{\mathrm{n}}$ \\
\hline 3 & 6 & 8 & 4 \\
\hline 4 & 24 & 16 & 10 \\
\hline 5 & 120 & 32 & 26 \\
\hline 6 & 720 & 64 & 76 \\
\hline
\end{tabular}

Table 7. Transformation of 8-length Walsh code set generated using the permutation $\{1,2,3,4\}$ into Walsh - Hadamard orderings

\begin{tabular}{|c|c|c|c|c|c|c|}
\hline $\begin{array}{l}\text { 8-length Walsh } \\
\text { code Set } \\
\text { constructed using } \\
\text { the permutation } \\
\{1,2,3,4\}\end{array}$ & $\begin{array}{l}\text { 3-bit Gray } \\
\text { code using } \\
\text { permutatio } \\
\mathrm{n}\{3,2,1\}\end{array}$ & $\begin{array}{l}\text { Natural Binary } \\
\text { Order } \\
\text { Sequence }\end{array}$ & $\begin{array}{l}\text { Gray } \\
\text { rank }\end{array}$ & $\begin{array}{l}\text { Rearranged } \\
\text { 8-length Walsh code set } \\
\text { Walsh-Hadamard } \\
\text { Sequence }\end{array}$ & $\begin{array}{l}\text { Row Transition } \\
\text { Count } \\
\left(\mathrm{TC}_{\mathrm{R}}\right)\end{array}$ & $\begin{array}{l}\text { Column } \\
\text { Count } \\
\left(\mathrm{TC}_{\mathrm{C}}\right)\end{array}$ \\
\hline 00000000 & 000 & 0 & 0 & 00000000 & 0 & 0 \\
\hline 00011110 & 100 & 1 & 7 & 01010101 & 7 & 7 \\
\hline 00110011 & 110 & 2 & 3 & 00110011 & 3 & 3 \\
\hline 00101101 & 010 & 3 & 4 & 01100110 & 4 & 4 \\
\hline 01100110 & 011 & 4 & 1 & 00001111 & 1 & 1 \\
\hline 01111000 & 111 & 5 & 6 & 01011010 & 6 & 6 \\
\hline 01010101 & 101 & 6 & 2 & 00111100 & 2 & 2 \\
\hline 01001011 & 001 & 7 & 5 & 01101001 & 5 & 5 \\
\hline
\end{tabular}

Table 8. Transformation of 8-length Walsh code set generated using the permutation $\{1,2,3,4\}$ into Walsh-Paley Sequence

\begin{tabular}{|c|c|c|c|c|c|c|}
\hline \multicolumn{7}{|c|}{ orderings } \\
\hline $\begin{array}{l}\text { 8-length Walsh } \\
\text { code Set } \\
\text { constructed using } \\
\text { the permutation } \\
\{1,2,3,4\}\end{array}$ & $\begin{array}{c}\text { 3-bit Gray } \\
\text { code Using } \\
\text { permutation } \\
\{1,2,3\}\end{array}$ & $\begin{array}{l}\text { Natural Binary } \\
\text { Order } \\
\text { Sequence }\end{array}$ & $\begin{array}{l}\text { Gray } \\
\text { rank }\end{array}$ & $\begin{array}{c}\text { Rearranged } \\
\text { 8-length } \\
\text { Walsh code set } \\
\text { Walsh-Paley Sequence }\end{array}$ & $\begin{array}{c}\text { Row Transition } \\
\text { Count } \\
\left(\mathrm{TC}_{\mathrm{R}}\right)\end{array}$ & $\begin{array}{c}\text { Column Transition } \\
\text { Count } \\
\left(\mathrm{TC}_{\mathrm{C}}\right)\end{array}$ \\
\hline 00000000 & 000 & 0 & 0 & 00000000 & 0 & 0 \\
\hline 00011110 & 001 & 1 & 1 & 00001111 & 1 & 1 \\
\hline 00110011 & 011 & 2 & 3 & 00110011 & 3 & 3 \\
\hline 00101101 & 010 & 3 & 2 & 00111100 & 2 & 2 \\
\hline 01100110 & 110 & 4 & 7 & 01010101 & 7 & 7 \\
\hline 01111000 & 111 & 5 & 6 & 01011010 & 6 & 6 \\
\hline 01010101 & 101 & 6 & 4 & 01100110 & 4 & 4 \\
\hline 01001011 & 100 & 7 & 5 & 01101001 & 5 & 5 \\
\hline
\end{tabular}

rows and columns is obtained for few permutations using the mapping technique. Walsh - Hadamard matrix ordering is obtained by Gray-Binary mapping with the permutation $\{3,2,1\}$ and Walsh-Paley matrix can be generated using the permutation $\{1,2,3\}$. Possibility of Walsh code set generation in strict sequency, Harmuth, Haar and other orderings is to be investigated. Future work includes the generation of different Walsh-like code sets of any length using Gray and Inverse Gray codes with any integer value of ' $n$ '.

\section{References}

1. Beauchamp KG (1975) Walsh functions and their applications. London: Acad. Press.

2. Duc-Minh Pham, Premkumar AB and Madhukumar AS (2011) Error detection and correction in communication channels using Inverse Gray RSNS codes. IEEE Trans. Comm. 59 (4). 975-986.

3. Falkowski BJ and Sasao T (2005) Unified algorithm to generate Walsh functions in four different orderings IEE Proc. visual ISP.152 (6), 819-826.

4. Harmuth HF (1969) Applications of Walsh functions in communications. IEEE Spectrum. 6, 82-91.

5. Jaya Sankar K (2004) Development of algorithms for a certain combinatorial optimization problem, PhD. Thesis. Osmania Univ.

6. Robert W Doran (2007) The Gray code. JUCS. Vol.13, no.11, 1573-1597.

7. Walsh JL (1923) A closed set of normal orthogonal functions. AJM. Vol. 45. 5-24. 